\title{
LINC00473 exacerbates osteoarthritis development by promoting chondrocyte apoptosis and proinflammatory cytokine production through the miR-424-5p/LY6E axis
}

\author{
GUIYONG FAN $^{1}$, JINLIAN LIU ${ }^{1}$, YESONG ZHANG $^{1}$ and XINXIAN GUAN ${ }^{2}$ \\ ${ }^{1}$ Department of Orthopedics, Suzhou Kowloon Hospital, Shanghai Jiangtong University \\ School of Medicine, Suzhou, Jiangsu 215028; ${ }^{2}$ Department of Neonatology, \\ Children's Hospital of Soochow University, Suzhou, Jiangsu 215025, P.R. China
}

Received September 25, 2020; Accepted May 28, 2021

DOI: $10.3892 /$ etm.2021.10682

\begin{abstract}
Osteoarthritis (OA) is a common degenerative joint disease that has been identified as one of the major health burdens in aging individuals. Long non-coding RNAs (lncRNAs) participate in the development of diverse diseases, including OA. Among them, lncRNA long intergenic non-protein coding RNA 473 (LINC00473) is one of the few upregulated lncRNAs. The present study aimed to explore the role of LINC00473 and its regulatory mechanism in OA development. Flow cytometry analyses and ELISA were carried out to detect chondrocyte apoptosis and the concentration of proinflammatory cytokines, respectively. The results suggested that LINC00473 knockdown significantly reduced chondrocyte apoptosis and the production of proinflammatory cytokines in IL-1 $\beta$-stimulated C28/I2 cells compared with transfection with small interfering RNA-negative control (si-NC). Western blot analyses were performed to examine protein levels of apoptotic markers (caspase-3, Bax and Bcl-2) in C28/I 2 cells. Subsequently, an OA rat model was established to explore the role of LINC00473 in vivo. The results indicated that, compared with the $\mathrm{OA}+$ adeno-associated virus si-NC group, LINC00473 knockdown significantly suppressed the degradation of chondrocyte extracellular matrix and the production of proinflammatory cytokines in OA model rats. Furthermore, bioinformatics analysis, luciferase reporter and RNA immunoprecipitation assays indicated that LINC00473 served as a microRNA (miR)-424-5p sponge in C28/I2 cells, and that lymphocyte antigen 6 locus E (LY6E) was the downstream target. In addition, the inhibitory effects of LINC00473 knockdown on chondrocyte apoptosis and the inflammatory
\end{abstract}

Correspondence to: Dr Xinxian Guan, Department of Neonatology, Children's Hospital of Soochow University, 92 Zhongnan Street, Suzhou, Jiangsu 215025, P.R. China

E-mail: guanxinxian@hotmail.com

Key words: long intergenic non-protein coding RNA 473, microRNA-424-5p, lymphocyte antigen 6 locus E, osteoarthritis response could be reversed by LY6E overexpression in IL-1 $\beta$-stimulated C28/I2 cells. In summary, the findings indicated that LINC00473 contributed to OA progression by modulating the miR-424-5p/LY6E axis, which may serve as a potential therapeutic strategy for patients with OA.

\section{Introduction}

As a chronic joint disease, osteoarthritis (OA) has seriously threatened the health of millions of individuals globally and has led to disability (1). Multiple factors are considered to be involved in the etiology of OA, including intracellular metabolism, inflammation and extracellular matrix (ECM) degeneration (2-4). Moreover, obesity and aging increase the risk of developing OA (5). The current treatments for OA, such as drug, electromagnetic therapy and acupuncture therapy, are mainly aimed at pain relief (6-8), thus OA remains difficult to cure. Further investigation of the pathogenesis of $\mathrm{OA}$ is required to elucidate effective therapies to prevent $\mathrm{OA}$ and reduce its severity.

Long non-coding RNAs (lncRNAs) are RNA molecules with $>200$ nucleotides that do not encode proteins (9). A multitude of studies have manifested that lncRNAs are involved in multiple diseases (10-12). For example, IncRNA AT-rich interactive domain 2-IR aggravates renal inflammation by activating the IL- $1 \beta$-induced $N F-\kappa B$ signaling pathway, as well as inducing the production of inflammatory cytokines (13). LncRNA urothelial cancer-associated 1 promotes the development of Parkinson's disease by aggravating the inflammatory response and exacerbating the damage of dopaminergic neurons (14). LncRNA metastasis-associated lung adenocarcinoma transcript 1 (MALAT1) inhibits ECM degradation and cell apoptosis by targeting miR-150-5p/AKT3 axis (15). Moreover, previous studies have revealed that long intergenic non-protein coding RNA 473 (LINC00473) serves a notable role in various diseases. For example, LINC00473 has been identified as a notable biomarker in ischemic stroke through the subpathway-LNCE method, which was designed to identify lncRNAs that competitively modulated functions in diseases (16). LINC00473 downregulation contributes to the development of preeclampsia (17). Additionally, a previous 
study demonstrated that LINC00473 was highly expressed in patients with severe pain associated with OA (18). However, the importance of LINC00473 in OA pathology requires further elucidation.

It has been proposed that competing endogenous RNAs (ceRNAs) could function as molecular sponges for microRNAs (miRNAs/miRs) and then modulate the expression of target genes (19). In previous years, an increasing number of studies have demonstrated that lncRNAs function as ceRNAs during the pathogenesis of multiple diseases, including OA. For example, upregulation of lncRNA RNA component of mitochondrial RNA processing endoribonuclease aggravates the development of coronary heart disease via the miR-206/autophagy-related 3 axis (20). LncRNA MALAT1 facilitates renal tubular epithelial pyroptosis by sponging miR-23c and targeting ELAV-like protein 1 in diabetic nephropathy (21). LncRNA small nucleolar RNA host gene 5 promotes chondrocyte proliferation by targeting the miR-26a/sex determining region Y-box 2 axis in OA (22). Additionally, recent research has indicated that LINC00473 may participate in OA pathogenesis via dysregulated ceRNA interactions (18). However, the specific mechanism of LINC00473 in OA has not been fully explored. The present study aimed to investigate the effects of LINC00473 on the pathogenesis of OA.

\section{Materials and methods}

Clinical samples. OA cartilage tissues $(n=24)$ were obtained from 24 patients (11 males and 13 females; age, 51-70 years) undergoing total knee replacement surgery and 24 healthy cartilage tissue samples were collected from patients (10 males and 14 females; age, 33-42 years) undergoing traumatic amputations at the Children's Hospital of Soochow University (Jiangsu, China) between January 2014 and December 2019. Additionally, normal cartilage tissues $(n=24)$ were collected from donors who underwent amputation with no diagnostic history of rheumatoid arthritis or OA at the same hospital. All donors signed informed consent forms. The present study was approved by The Human Ethics Committee of Children's Hospital of Soochow University. All tissue samples were stored at $-80^{\circ} \mathrm{C}$. Inclusion criteria were listed as follows: i) Patients who met clinical manifestations of knee osteoarthritis (23); ii) patients with complete medical records; iii) patients who had not received any antibiotic treatment within 3 months before admission; iv) patients who were willing to cooperate and participate in the investigation. Exclusion criteria were also listed as follows: i) Patients with tumors, organ dysfunction, infectious diseases, autoimmune diseases, neurological disorders, cardiovascular diseases or cerebrovascular diseases; ii) patients who were allergic to drugs used in the present study; iii) patients with physical disabilities and unable to take care of themselves; iv) patients who were transferred to other hospitals.

Rat model of $O A$. The animal experiments were approved by The Attitude of the Animal Care \& Welfare Committee of Children's Hospital of Soochow University (permit no. 2019-044). A total of 20 male rats (age, 6 weeks; weight, 230-260 g; Beijing Vital River Laboratory Animal Technology Co., Ltd.) were randomly divided into the following four groups ( $\mathrm{n}=5$ rats/group) for subsequent experiments: i) Sham; ii) $\mathrm{OA}$; iii) $\mathrm{OA}+$ adeno-associated virus (AAV) small interfering RNA (si/siRNA)-negative control (NC); and iv) and $\mathrm{OA}+\mathrm{AAV}$ si-LINC00473. All procedures were conducted following recommendations of The Guide for the Care and Use of Laboratory Animals (24).

The OA rat model was established by anterior cruciate ligament (ACL) transection and partial medial meniscectomy. In brief, after anesthetization by the intraperitoneal injection of pentobarbital (35 mg/kg weight), a 1-cm longitudinal incision on the medial side of the right knee joint was made and the medial ligament was exposed. After the ACL and medial meniscus were removed, the joint capsule and the skin was sutured and bandaged under a sterile environment. For the sham group, the same incisions were made in rats but without meniscus or ACL resection. For AAV-injected groups, OA model rats were intra-articularly injected with recombinant (r) AAV5 expressing si-NC or si-LINC00473 (1.65×10 ${ }^{10} \mathrm{vg} /$ joint $)$ using 33-gauge needles and 25- $\mu$ l gastight syringes (both Hamilton Company) after modeling. rAAV5 was obtained from Han heng Biotechnology (Shanghai) Co., Ltd. All groups of rats (sham, OA model and AAV injected) were kept in separate cages and provided with 12/12 h light/dark cycle and ad libitum food and water. Humidity was $50-55 \%$ and feeding temperature was set to $20-23^{\circ} \mathrm{C}$. At 4 weeks after surgery, under intraperitoneal anesthesia using $1,000 \mathrm{mg} / \mathrm{kg}$ urethane, rats were sacrificed by cervical dislocation and death was confirmed by absence of corneal reflex, spontaneous breathing and heartbeat for a period of $>5 \mathrm{~min}$. The cartilage tissues of rats in each group were collected for subsequent experiments.

Cell culture and treatment. Human cartilage C28/I2 cells were provided by American Type Culture Collection. The articular cartilage specimens of rats were minced into small sections and then digested with $0.2 \%$ type II collagenase (MilliporeSigma; Merck KGaA) in DMEM (Gibco; Thermo Fisher Scientific, Inc.) at $37^{\circ} \mathrm{C}$ for 5-6 h. To separate the rat chondrocytes, the cell suspension was centrifuged at $12,000 \mathrm{x} \mathrm{g}$ for $10 \mathrm{~min}$ at $4^{\circ} \mathrm{C}$. C28/I2 cells and rat chondrocytes were incubated in DMEM (Gibco; Thermo Fisher Scientific, Inc.) with $10 \%$ FBS, $0.1 \mathrm{mg} / \mathrm{ml}$ streptomycin and $100 \mathrm{U} / \mathrm{ml}$ penicillin (Gibco; Thermo Fisher Scientific, Inc.) at $37^{\circ} \mathrm{C}$ with $5 \% \mathrm{CO}_{2}$. To induce the pathological condition of OA in cells, C28/I2 cells were treated with $10 \mathrm{ng} / \mathrm{ml}$ IL-1 $\beta$ (Sigma-Aldrich; Merck KGaA) for $24 \mathrm{~h}$ at $37^{\circ} \mathrm{C}$. Non-treated cells served as a control.

$R N A$ extraction and reverse transcription-quantitative $P C R$ (RT-qPCR). Total RNA was extracted from the cartilage tissues of patients with OA, control donors, cultured C28/I2 cells and the cartilage tissues of rats in each group using TRIzol ${ }^{\circledR}$ reagent (Invitrogen; Thermo Fisher Scientific, Inc.). High-Capacity cDNA Reverse Transcription kit (Applied Biosystems; Thermo Fisher Scientific, Inc.) was used to reverse transcribe total RNA to cDNA according to the manufacturer's recommendations. After reverse transcription, $\mathrm{SYBR}^{\circledR}$ Green Mix (Takara Bio, Inc.) was used for qPCR with the Biosystems 7300 Real-Time PCR system (Applied Biosystems; Thermo Fisher Scientific, Inc.). The qPCR was conducted at $95^{\circ} \mathrm{C}$ for $10 \mathrm{~min}$ followed by 40 cycles of $95^{\circ} \mathrm{C}$ for $30 \mathrm{sec}$ and $60^{\circ} \mathrm{C}$ for $1 \mathrm{~min}$. The $2^{-\Delta \Delta \mathrm{Ct}}$ method was applied to calculate the relative 
Table I. Sequences of primers used for reverse transcription-quantitative PCR.

\begin{tabular}{|c|c|}
\hline Gene & Sequence $\left(5^{\prime} \rightarrow 3^{\prime}\right)$ \\
\hline \multirow[t]{2}{*}{ LINC00473 } & F: GGGAGCTTGAGCTGAGATGG \\
\hline & R: TTCGCAGTTTCCTAGTGGGAC \\
\hline \multirow[t]{2}{*}{ miR-424-5p } & F: CAGCAGCAATTCATGTTTTGAA \\
\hline & R: CTCTACAGCTATATTGCCAGCCAC \\
\hline \multirow{2}{*}{ miR-15b-5p } & F: TAGCAGCACATCATGGTTTACA \\
\hline & R: CTCTACAGCTATATTGCCAGCCAC \\
\hline \multirow[t]{2}{*}{ miR-497-5p } & F: CAGCAGCACACTGTGGTTTG \\
\hline & R: CTCTACAGCTATATTGCCAGCCAC \\
\hline \multirow[t]{2}{*}{$\operatorname{miR}-16-5 p$} & F: TAGCAGCACGTAAATATTGGCG \\
\hline & R: CTCTACAGCTATATTGCCAGCCAC \\
\hline \multirow[t]{2}{*}{ miR-6838-5p } & F: TAATCTCAGCTGGCAACTGTG \\
\hline & R: CTCTACAGCTATATTGCCAGCCAC \\
\hline \multirow[t]{2}{*}{ miR-195-5p } & F: TAGCAGCACAGAAATATTGGCG \\
\hline & R: CTCTACAGCTATATTGCCAGCCAC \\
\hline \multirow[t]{2}{*}{$\operatorname{miR}-15 a-5 p$} & F: TAGCAGCACATAATGGTTTGTGC \\
\hline & R: CTCTACAGCTATATTGCCAGCCAC \\
\hline \multirow[t]{2}{*}{ LY6E } & F: CTGCGTGACTGTGTCTGCTA \\
\hline & R: CCTGCATGGGAAATGAGGCT \\
\hline \multirow[t]{2}{*}{ GAPDH } & F: GAAGGTGAAGGTCGGAGTC \\
\hline & R: GAAGATGGTGATGGGATTTC \\
\hline \multirow[t]{2}{*}{ U6 } & F: GCTTCGGCAGCACATATACTAAAAT \\
\hline & R: CGCTTCACGAATTTGCGTGTCAT \\
\hline
\end{tabular}

LINC00473, long intergenic non-protein coding RNA 473; miR, microRNA; LY6E, lymphocyte antigen 6 locus E; F, forward; $\mathrm{R}$, reverse.

gene expression (25). Internal references were U6 and GAPDH. The relative primer sequences are provided in Table I.

Cell transfection. Small interfering RNA (siRNA) against LINC00473 (si-LINC00473) and lymphocyte antigen 6 locus E (LY6E; si-LY6E) with si-negative control (NC) as the control and pcDNA3.1/LY6E with empty pcDNA3.1 vector as the control were synthesized by Shanghai GenePharma Co., Ltd. miR-424-5p mimics, miR-424-5p inhibitor and their corresponding controls (NC mimics and NC inhibitor) were purchased from Shanghai GenePharma Co., Ltd. Lipofectamine $2000^{\circledR}$ (Invitrogen; Thermo Fisher Scientific, Inc.) was utilized to transfect these plasmids or vectors into normal or IL-1 $\beta$-stimulated C28/I 2 cells $\left(5 \times 10^{6}\right.$ cells/well) for $48 \mathrm{~h}$ following the manufacturer's instructions. The concentration of miR-424-5p mimics/inhibitors or $\mathrm{NC}$ mimics/inhibitors was $50 \mathrm{nM}$, and that of siRNAs and vectors were 40 and $10 \mathrm{nM}$, respectively. The transfection efficiency was examined by RT-qPCR after $48 \mathrm{~h}$. The sequences of all molecules used for cell transfection are presented in Table II.

Fluorescence in situ hybridization (FISH). LINC00473 probes were designed and synthesized by Guangzhou RiboBio Co., Ltd. Probe signals were detected using an RNA FISH Kit (Guangzhou RiboBio Co., Ltd.) following the manufacturer's protocols. In brief, IL-1 $\beta$-stimulated C28/I2 cells were fixed in $4 \%$ formalin for $10 \mathrm{~min}$ at room temperature. After permeabilization in PBS with $0.5 \%$ Triton $\mathrm{X}-100$ at $4^{\circ} \mathrm{C}$ for $20 \mathrm{~min}$, cells were dehydrated using $100 \%$ ethanol for $15 \mathrm{~min}$ at room temperature. Subsequently, cells were incubated in $10 \mu \mathrm{l}$ hybridization solution $(900 \mathrm{mM}$ NaCI, $20 \mathrm{mM}$ Tris/HCI, $20 \%$ formamide, distilled $\mathrm{H}_{2} \mathrm{O}$ and $0.01 \%$ SDS) at $37^{\circ} \mathrm{C}$ for $30 \mathrm{~min}$ and then further incubated with LINC00473 probe $(300 \mathrm{ng} / \mathrm{ml})$ overnight at $4^{\circ} \mathrm{C}$. Afterwards, cells were washed twice with saline sodium for $10 \mathrm{~min}$ at $25^{\circ} \mathrm{C}$. The slides were incubated with tyramide signal amplification (TSA) fluorescent signal reaction solution (cat. no. NEL701001KT; PerkinElmer, Inc.) for $30 \mathrm{~min}$ and counterstained with DAPI (Beyotime Institute of Biotechnology) at room temperature for $5 \mathrm{~min}$ following the manufacturer's protocol. Fluorescence microscopy (Leica Microsystems $\mathrm{GmbH}$ ) was used to capture images at x200 magnification.

Luciferase reporter assay. The binding sites between LINC00473 and miR-424-5p were predicted using starBase version 3.0 (http://starbase.sysu.edu.cn/). The sequences of LINC00473 were cloned to the pmirGLO reporter plasmids (Promega Corporation) to form wild-type (Wt; pmirGLO-LINC00473-Wt-1/2) LINC00473. Mutated (Mut; pmirGLO-LINC00473-Mut-1/2) LINC00473 luciferase vectors were constructed using Phusion Site-Directed Mutagenesis Kit (Thermo Fisher Scientific,Inc.). The pmirGLO luciferase reporter vectors of the LY6E 3'untranslated region (3'UTR) were obtained in the same way. C28/I2 cells were seeded into 24 -well plates $\left(5 \times 10^{4}\right.$ cells/well) at $37^{\circ} \mathrm{C}$ overnight. Subsequently, in the first test, the pmirGLO-LINC00473-Wt-1/2 or pmirGLO-LINC00473-Mut-1/2 vectors were co-transfected with NC mimics or miR-424-5p mimics into C28/I2 cells using Lipofectamine ${ }^{\circledR} 3000$ (Invitrogen; Thermo Fisher Scientific, Inc.). In the second test, the pmirGLO-LY6E 3'UTR-Wt or pmirGLO-LY6E 3'UTR-Mut vectors (Generay Biotech Co., Ltd.) were co-transfected with $\mathrm{NC}$ mimics or miR-424-5p mimics into C28/I2 cells using Lipofectamine ${ }^{\circledR}$ 3000 (Invitrogen; Thermo Fisher Scientific, Inc.). The concentration of plasmids was $50 \mathrm{ng} / \mathrm{well}$ and that of miR-424-5p mimics or NC mimics was $20 \mathrm{nM}$. After $48 \mathrm{~h}$ of transfection, the Dual-Luciferase Reporter Assay system (Promega Corporation) was used to detect the luciferase activity. The firefly luciferase activity was normalized to that of Renilla luciferase.

RNA immunoprecipitation (RIP) assay. The RIP assay was performed using the EZ-Magna RIP Kit (MilliporeSigma) following the manufacturer's recommendations. In brief, C28/I2 cells were lysed in RIP lysis buffer (MilliporeSigma) containing protease inhibitor cocktail and RNase inhibitor for $5 \mathrm{~min}$ of incubation on ice followed by centrifugation at $10,000 \mathrm{x}$ g for $10 \mathrm{~min}$ at $4^{\circ} \mathrm{C}$. Cell lysate was incubated with RIP buffer containing Protein $\mathrm{G}$ magnetic beads (cat. no. 88848; Thermo Fisher Scientific, Inc.) coated with Ago2 antibody (1:50; cat. no. ab186733; Abcam) or the control IgG antibody (1:1,000; cat. no. ab6702; Abcam) overnight at $4^{\circ} \mathrm{C}$ according to the manufacturer's recommendations. Next, the magnetic beads were washed three times using RIP wash buffer and 
Table II. Sequences of molecules used for cell transfection.

\begin{tabular}{llc}
\hline Molecule & \multicolumn{1}{c}{ Sense (5'-3') } & Antisense (5'-3') \\
\hline si-LINC00473 & CAAGCACUCAUGUUCUAAAGA & UUUAGAACAUGAGUGCUUGUG \\
si-NC (for si-LINC00473) & GGACCGCUAAUCUAAUCAAUA & UAUUGAUUAGAUUAGCGGUCC \\
si-LY6E & GCUUGAACCAGAAGAGCAAUC & UUGCUCUUCUGGUUCAAGCAG \\
si-NC (for si-LY6E) & AACGACCAUGAGAGUAGUCAC & GUGACUACUCUCAUGGUCGUU \\
miR-424-5p inhibitor & UUCAAAACAUGAAUUGCUGCUG & - \\
NC inhibitor & CAGUACUUUUGUGUAGCACAAA & - \\
miR-424-5p mimics & CAGCAGCAAUUCAUGUUUUGAA & - \\
NC mimics & CAAAACAUGAAUUGCUGCUGUU & - \\
\hline
\end{tabular}

NC, negative control; miR, microRNA; si, small interfering.

treated with proteinase $\mathrm{K}$ (Thermo Fisher Scientific, Inc.) at $55^{\circ} \mathrm{C}$ for $30 \mathrm{~min}$. Finally, the co-precipitated RNAs were measured using RT-qPCR.

Hematoxylin and eosin $(H \& E)$ staining assay. The cartilage tissues from rats in the sham or OA groups were fixed with 4\% paraformaldehyde (Wuhan Boster Biological Technology, Ltd.) at room temperature for $24 \mathrm{~h}$, embedded in paraffin, cut into $4-\mu \mathrm{m}$ thick sections and visualized under light microscope (Leica Microsystems $\mathrm{GmbH}$ ) at x200 magnification after $H \& E$ staining.

Flow cytometry analysis. Early and late apoptosis was evaluated by flow cytometry using the Annexin V-Fluorescein Isothiocyanate (FITC) Apoptosis Detection kit (MilliporeSigma). Briefly, transfected C28/I2 cells $\left(1 \times 10^{5}\right.$ cells/well) were washed twice with cold PBS and resuspended in binding buffer. Next, cells were incubated with Annexin V-FITC and propidium iodide in the dark at room temperature for $10 \mathrm{~min}$. Stained cells were identified by a FACScalibur flow cytometer (Becton, Dickinson and Company) analyzed using FlowJo v.10 (FlowJo LLC).

Western blotting. Proteins were extracted from cartilage tissues of rats in each group and cultured C28/I2 cells using RIPA lysis buffer (Beyotime Institute of Biotechnology) containing phenylmethylsulfonyl fluoride. The protein concentration was quantified using a Bicinchoninic Acid Protein Assay kit (Beyotime Institute of Biotechnology). After $25 \mu \mathrm{g}$ protein/lane was separated by $10 \%$ SDS-PAGE, proteins were transferred onto PVDF membranes (MilliporeSigma). Next, the membranes were blocked at room temperature for $2 \mathrm{~h}$ with $5 \%$ non-fat milk and then incubated overnight at $4^{\circ} \mathrm{C}$ with primary antibodies (all Abcam) targeted against the following: Caspase- 3 (cat. no. ab13847; 1:500), Bax (cat. no. ab32503; 1:1,000), Bcl-2 (cat. no. ab196495; 1:1,000), $\beta$-actin (cat. no. ab8227; 1:1,000), collagen II (cat. no. ab239007; 1:5,000), MMP1 (cat. no. ab134184; 1:1,000), MMP13 (cat. no. ab39012; 1:3,000) and LY6E (cat. no. ab201098; 1:1,000). $\beta$-actin was used as the internal control. After washing three times with TBST containing $0.1 \%$ Tween 20 , the membranes were further incubated with goat anti-rabbit IgG H\&L (HRP) secondary antibodies (cat. no. ab97051; 1:10,000; Abcam) at room temperature for $2 \mathrm{~h}$. Finally, the immunoreactive bands were visualized using an ECL chemiluminescent detection system (Thermo Fisher Scientific, Inc.) and GeneGenius Gel Light Imaging system (Syngene) and analyzed using ImageJ software (version 1.49; National Institutes of Health).

ELISA. Blood was collected from the rats of each group and sera was obtained after centrifugation at $6,000 \mathrm{xg}$ for $15 \mathrm{~min}$ at $4^{\circ} \mathrm{C}$. The concentrations of inflammatory cytokines, including IL-6 (cat. no. ab234570; Abcam), IL-8 (cat. no. SEKR-0071; Solarbio) and TNF- $\alpha$ (cat. no. ab236712; Abcam), were measured using ELISA kits according to the manufacturer's protocols.

Statistical analysis. All experiments were repeated three times independently. Experimental data are expressed as the mean \pm standard deviation (unless otherwise indicated). Statistical analyses were performed using SPSS v19.0 software (IBM Corp.). The independent Student's t-test was used to analyze the significant differences between two groups. Statistical significance among three or more groups was assessed using one-way ANOVA followed by Tukey's post hoc test. $\mathrm{P}<0.05$ was considered to indicate a statistically significant difference.

Bioinformatics analysis. Seven candidate miRNAs (miR-424-5p, miR-15b-5p, miR-497-5p, miR-16-5p, miR-6838-5p, miR-195-5p and miR-15a-5p) were predicted using starBase v3.0 software (http://starbase.sysu.edu.cn/) under the condition of cross linking and immunoprecipitation-sequence data (high stringency $\geq 3$ ). The downstream target gene of miR-424-5p, LY6E, was also predicted from the starBase v3.0 website under the condition of degradome data (low strigency $\geq 1$ ) and the prediction program tools PITA, RNA22 and PicTar.

\section{Results}

LINC00473 is upregulated in cartilage tissues of patients with $O A$ and IL-1 $\beta$-treated chondrocytes. It has been previously reported that LINC00473 is one of the candidate 
biomarkers for OA (18). To explore the role of LINC00473 in the pathogenesis of OA, RT-qPCR analysis was conducted to examine LINC00473 expression levels in the cartilage tissues of patients with OA. As presented in Fig. 1A, LINC00473 expression was significantly upregulated in the cartilage tissues of patients with OA compared with individuals without OA. In accordance with previous studies $(26,27)$, the OA cell model was constructed using IL- $1 \beta$ to induce normal human chondrocytes C28/I2. The present study revealed that the expression level of LINC00473 was significantly higher in IL-1 $\beta$-stimulated C28/I2 cells compared with that in control C28/I2 cells (Fig. 1B).

LINC00473 knockdown reduces chondrocyte apoptosis and the inflammatory response in $I L-1 \beta$-treated C28/I2 cells. Apoptosis and inflammation serve notable roles in OA development (28). Based on the aforementioned results, the effects of LINC00473 on cell injury in OA were investigated. As presented in Fig. 2A, the expression level of LINC00473 was significantly decreased in C28/I2 cells transfected with si-LINC00473 compared with those transfected with si-NC. In the subsequent experiments, C28/I2 cells were treated with $10 \mathrm{ng} / \mathrm{ml} \mathrm{IL}-1 \beta$ to induce the pathological effects of OA, including apoptosis. The flow cytometry results revealed that LINC00473 knockdown significantly inhibited the apoptosis of IL-1 $\beta$-stimulated C28/I2 cells compared with the IL-1 $\beta+$ si-NC group (Fig. $2 \mathrm{~B}$ and C). Moreover, western blotting revealed that the protein levels of caspase-3 and Bax were significantly increased while Bcl-2 protein level was decreased after IL-1 $\beta$ treatment. Compared with the IL-1 $\beta+$ si-NC group, transfection of si-LINC00473 partially reversed IL-1 $\beta$-induced promotive or suppressive effect on protein levels of these apoptotic markers in C28/I2 cells (Fig. 2D and E). These data further indicated that LINC00473 knockdown decreased apoptosis in IL-1 $\beta$-treated C28/I2 cells. Increasing evidence has demonstrated that inflammation exacerbates the development of $\mathrm{OA}$ by producing excessive proinflammatory cytokines in chondrocytes (29-31). To verify whether LINC00473 influenced the release of proinflammatory cytokines, ELISAs were performed to determine the concentrations of IL-6, IL- 8 and TNF- $\alpha$ released by IL-1 $\beta$-treated C28/I2 cells. As presented in Fig. $2 \mathrm{~F}-\mathrm{H}$, the levels of these proinflammatory cytokines were significantly increased in C28/I 2 cells treated with IL-1 $\beta$ compared with these in the control group. Furthermore, these effects were partially inhibited by LINC00473 knockdown, as seen by the significant decrease in the levels of these proteins in the IL-1 $\beta+$ si-LINC00473 group compared with those in the IL-1 $\beta+$ si-NC group.

LINC00473 knockdown alleviates OA development in vivo. To further investigate the effect of LINC00473 on OA development in vivo, an OA model was successfully established in rats. $\mathrm{H} \& \mathrm{E}$ staining was conducted to determine the pathological alterations to the knee joint in OA model rats. As presented in Fig. 3A, sham rats presented a smoothed cartilage surface, no cartilage sclerosis and synovial hyperplasia. However, OA model rats manifested clear hyperplasia, sclerosis and joint wear. Subsequently, RT-qPCR was conducted to detect the expression of LINC00473 in vivo. As presented in Fig. 3B,
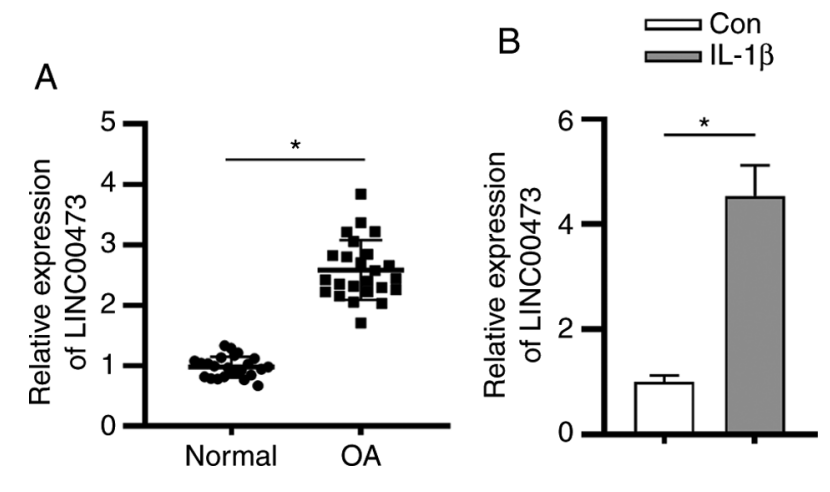

Figure 1. LINC00473 is upregulated in cartilage tissues of patients with OA and in IL-1 $\beta$-treated chondrocytes. (A) Expression of LINC00473 was measured in the cartilage tissues of patients with OA and healthy controls by reverse transcription-quantitative PCR analysis. (B) LINC00473 expression levels were detected in normal C28/I2 cells and in IL-1 $\beta$-treated C28/I2 cells. ${ }^{*} \mathrm{P}<0.05$. LINC00473, long intergenic non-protein coding RNA 473; OA, osteoarthritis; Con, control.

the expression levels of LINC00473 were significantly higher in the cartilage tissues of rats with OA compared with those of sham rats. Compared with the OA + AAV si-NC group, expression of LINC00473 was significantly decreased by the injection of AAV si-LINC00473. It has been previously reported that the degradation of ECM was closely associated with the destruction of joints in OA (32). To evaluate whether LINC00473 affected OA progression by promoting ECM degradation, the expression levels of collagen II, MMP1 and MMP13 in the cartilage tissue of rats in each group were measured (Fig. 3C and D). The data revealed that the protein expression levels of MMP1 and MMP13 were significantly upregulated in the cartilage tissues of OA model rats compared with the sham group. In contrast to the OA + AAV si-NC group, the injection of AAV si-LINC00473 significantly downregulated the protein expression levels of MMP1 and MMP13 in the cartilage tissues of rats with OA. However, the protein expression levels of collagen II indicated the opposite trend. In addition, it was revealed that the concentrations of proinflammatory cytokines, including IL-6, IL-8 and TNF- $\alpha$, were significantly increased in the cartilage tissues of OA model rats compared with sham rats. Moreover, LINC00473 knockdown in the OA + AAV si-LINC00473 group induced a significant decrease in proinflammatory cytokine concentrations in the cartilage tissues of rats with OA compared with the $\mathrm{OA}+$ AAV si-NC group (Fig. 3E-G).

LINC00473 acts as a sponge for miR-424-5p. Previous studies have indicated that lncRNAs participate in the pathogenesis of OA via the ceRNA pattern $(33,34)$. As a typical post-transcriptional mechanism, the ceRNA pattern refers to the lncRNAs that compete with mRNAs to bind with miRNAs, thus antagonizing the suppressive effects of miRNAs on mRNAs (35). As presented in Fig. 4A, LINC00473 was primarily localized in the cytoplasm of cells, indicating the post-transcriptional control of LINC00473 on gene expression in C28/I2 cells. To further investigate the potential mechanism of LINC00473 in C28/I2 cells, bioinformatics analysis was performed. According to the prediction of starBase, seven candidate miRNAs (miR-424-5p, miR-15b-5p, miR-497-5p, miR-16-5p, miR-6838-5p, miR-195-5p 
A

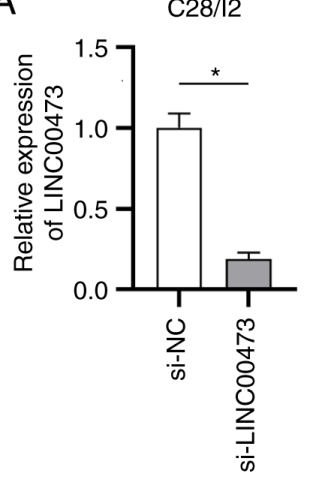

D

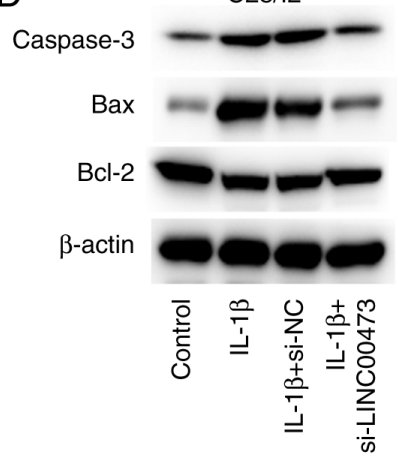

B

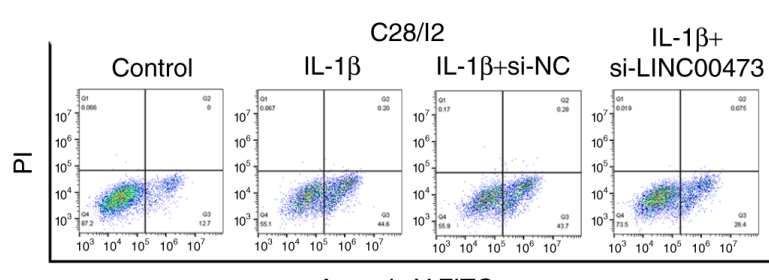

Annexin V-FITC

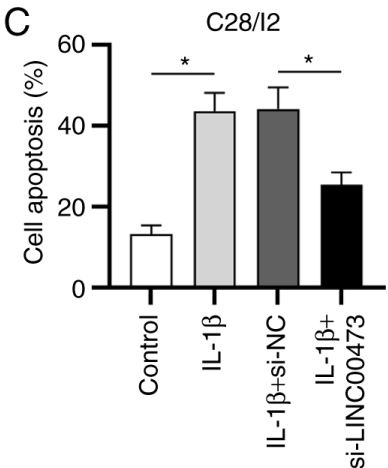

F

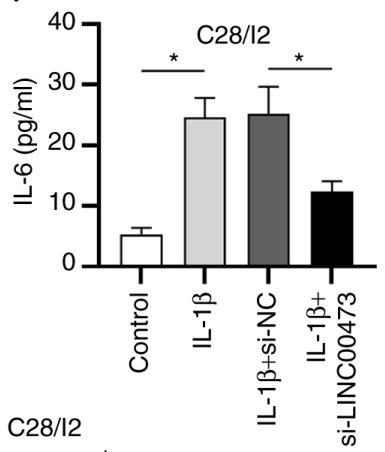

G

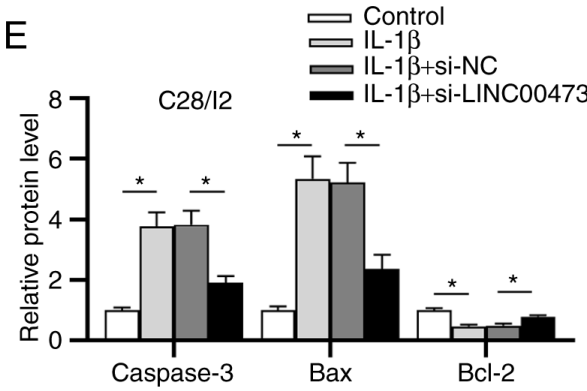

$\mathrm{H}$
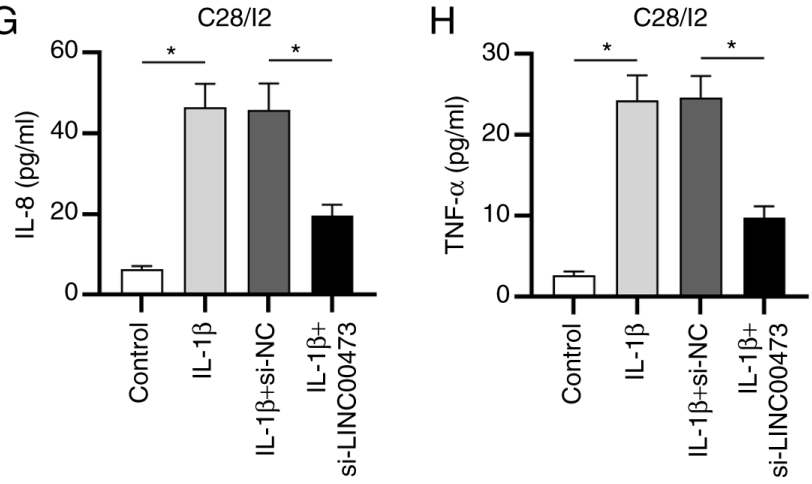

Figure 2. LINC00473 knockdown decreases chondrocyte apoptosis and the inflammatory response in IL-1 $\beta$-treated C28/I2 cells. (A) Knockdown efficacy of si-LINC00473 in C28/I2 cells was evaluated using reverse transcription-quantitative PCR analysis. Apoptosis of transfected C28/I2 cells was (B) determined using flow cytometry and (C) quantified. Expression levels of proapoptotic proteins (caspase-3 and Bax) and antiapoptotic protein Bcl-2 were analyzed in IL-1 $\beta$-treated C28/I2 cells using (D) western blotting and then (E) semi-quantified. Effects of LINC00473 knockdown on the production of proinflammatory cytokines (F) IL-6, (G) IL-8 and (H) TNF- $\alpha$ in IL-1 $\beta$-treated C28/I2 cells were measured using ELISAs. "P<0.05. LINC00473, long intergenic non-protein coding RNA 473; PI, propidium iodide; si, small interfering RNA; NC, negative control.

and miR-15a-5p) were revealed to share potential a binding site with LINC00473. Subsequently, RT-qPCR was performed to estimate the expressions of these candidate miRNAs, the results of which revealed that miR-424-5p demonstrated the most significant downregulation in IL-1 $\beta$-stimulated C28/I2 cells compared with the control cells (Fig. 4B). Whether LINC00473 interacted with miR-424-5p in C28/I2 cells was subsequently investigated. As presented in Fig. 4C, compared with the NC groups, miR-424-5p expression was significantly increased and decreased in C28/I2 cells following transfection with miR-424-5p mimics or miR-424-5p inhibitor, respectively. The two binding sites between LINC00473 and miR-424-5p were predicted using the starBase database (Fig. 4D). The luciferase reporter assay revealed that the luciferase activity of pmirGLO-LINC00473-Wt vectors at both site 1 and site 2 was significantly reduced by miR-424-5p mimics compared with the
NC mimic (Fig. 4E). By contrast, no difference was identified in the LINC00473-Mut-1/2 group between the NC mimics and miR-424-5p mimics groups, indicating that LINC00473 bound to miR-424-5p at the predicted sites in C28/I2 cells. The results implied that LINC00473 could bind with the two binding sites of miR-424-5p in C28/I2 cells. In addition, the RIP assay elucidated that LINC00473 and miR-424-5p were significantly enriched in RNA-induced silencing complexes immunoprecipitated by anti-Ago 2 compared with those immunoprecipitated by anti-IgG in C28/I2 cells (Fig. 4F).

LY6E is a direct target of miR-424-5p. Subsequently, the downstream target of miR-424-5p, i.e., LY6E was identified according to the prediction from the starBase website under the condition of degradome data Low strigency $(\geq 1)$ and the prediction program tools PITA, RNA22 and PicTar (miRNA-targets 


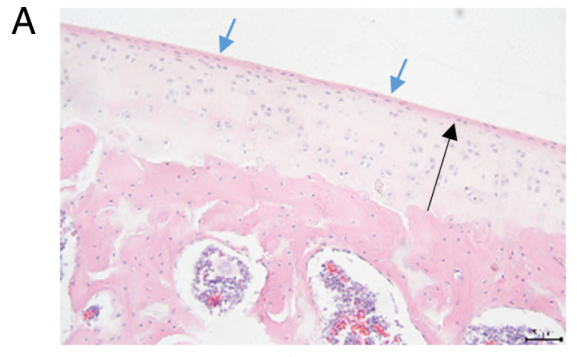

Sham

C

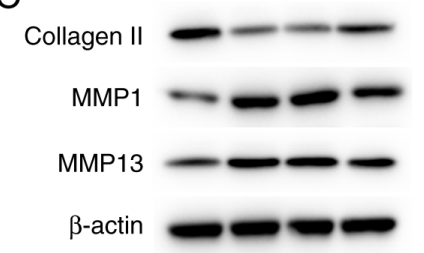

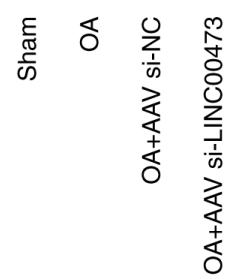

$\mathrm{F}$

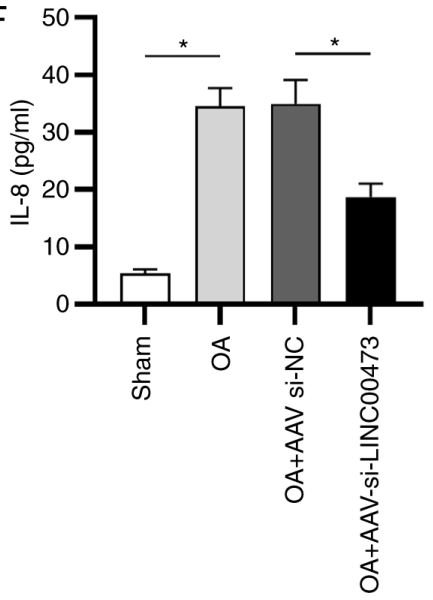

D

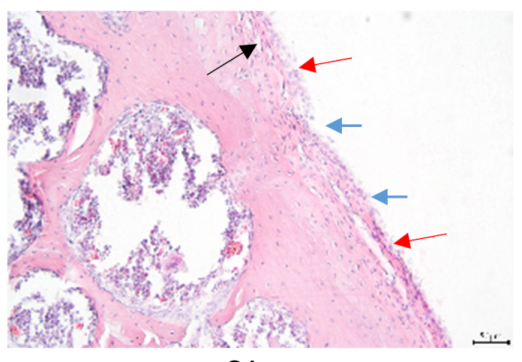

$\mathrm{OA}$
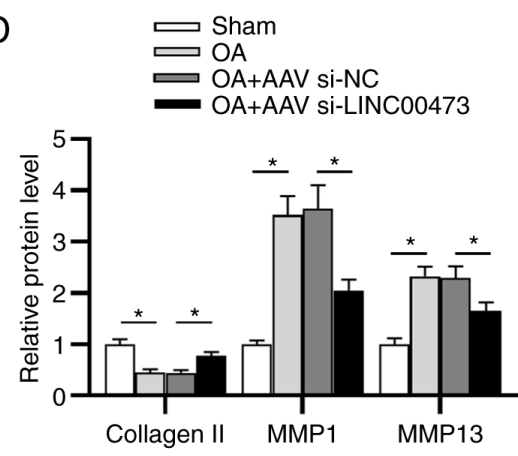

$\mathrm{E}$
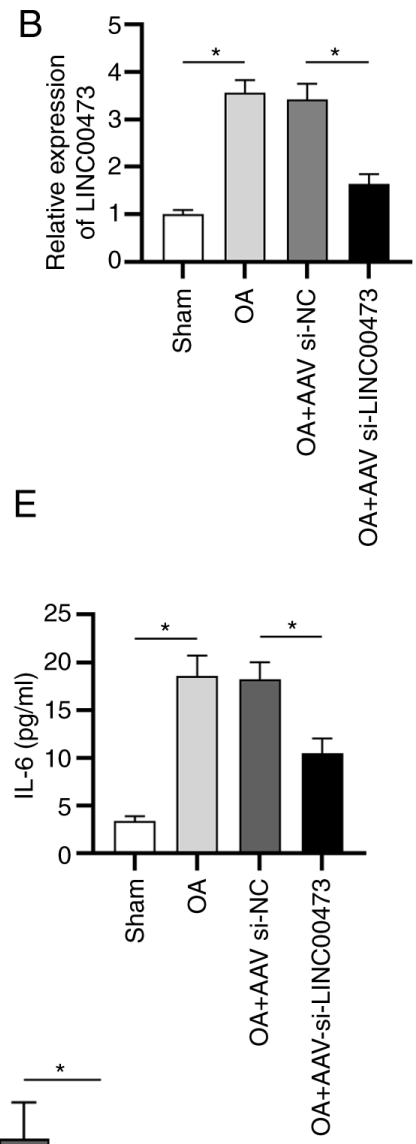

G

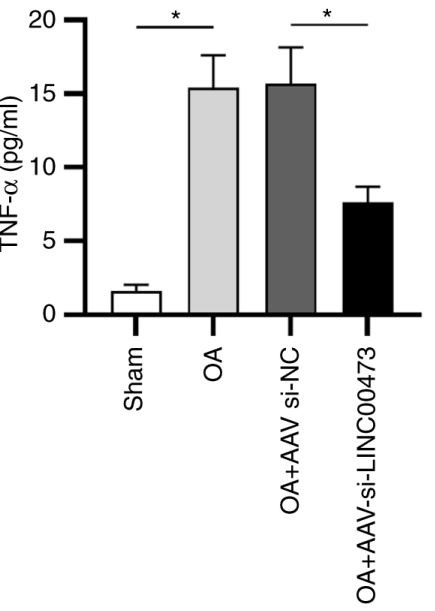

Figure 3. LINC00473 knockdown alleviates OA development in vivo. (A) Pathological changes in the knee joints of rats were observed using hematoxylin and eosin staining (magnification, x200; scale bar, $50 \mu \mathrm{m}$ ). Black arrow indicates thinning of articular cartilage tissue; blue arrow indicates the damage of cartilage surface and the existence of cartilage debris; red arrow indicates the formation of chondrocyte clusters around the bare cartilage. (B) Expression of LINC00473 was detected in rat cartilage tissues using reverse transcription-quantitative PCR. Protein expression levels of collagen II, MMP1 and MMP13 were (C) detected using western blotting and (D) semi-quantified after the injection of AAV si-LINC00473. ELISAs were used to determine the concentrations of proinflammatory cytokines (E) IL-6, (F) IL-8 and (G) TNF- $\alpha$ in the cartilage tissues of rats from each group. * P<0.05. LINC00473, long intergenic non-protein coding RNA 473; OA, osteoarthritis; MMP, matrix metallopeptidase; AAV, adeno-associated virus; si, small interfering RNA; NC, negative control.

predicting software). As determined by RT-qPCR, the expression of LY6E was significantly increased in C28/I2 cells stimulated with IL-1 $\beta$ compared with control cells (Fig. 5A). As demonstrated in Fig. 5B, miR-424-5p contained the binding site on the 3'UTR of LY6E in C28/I2 cells. The luciferase reporter assay revealed that the luciferase activity of the pmirGLO-LY6E 3'UTR-Wt vector was significantly decreased by miR-424-5p mimics transfection in C28/I2 cells compared with transfection with NC mimics. However, no difference was revealed in the luciferase activity of the pmirGLO-LY6E 3'UTR-Mut vector between the NC mimics and miR-424-5p mimics groups in C28/I2 cells (Fig. 5C). Additionally, both miR-424-5p and LY6E were revealed to have a significantly increased level of co-immunoprecipitation by Ago2 antibody compared with IgG antibody in C28/I2 cells (Fig. 5D). Moreover, the mRNA and protein expression levels of LY6E in C28/I2 cells were significantly decreased by miR-424-5p mimics transfection or LINC00473 knockdown compared 
A

LINC00473
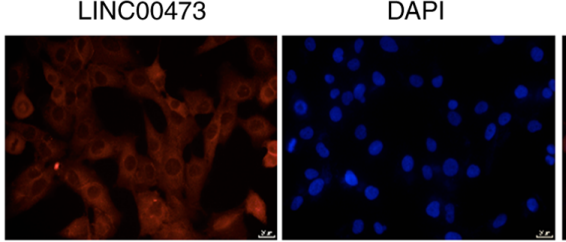

C

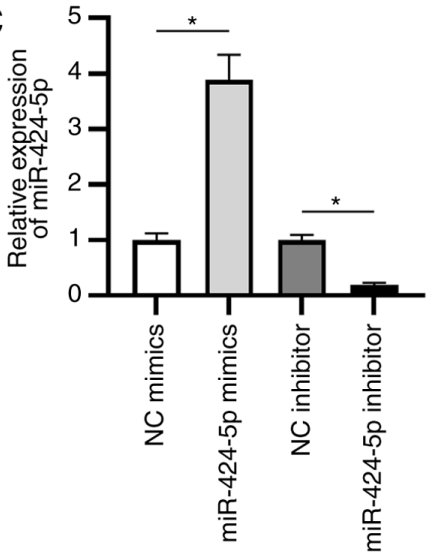

Merged

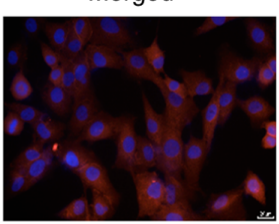

D

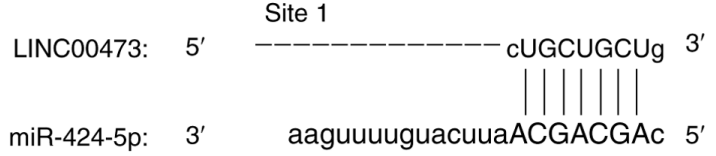

LINC00473: $\quad 5^{\prime} \quad$ guCUGAGUCCGAAGUUGCUGCUg $3^{\prime}$

miR-424-5p: $\quad 3^{\prime}$
|:|:: ||| ||||||||

$B$

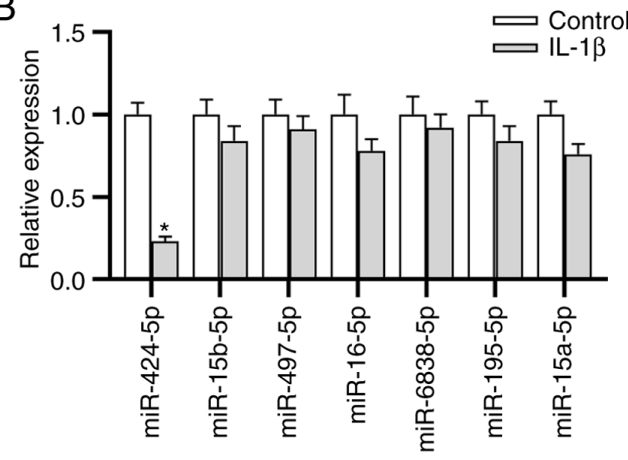

Site 1

aaGUUUUGUACUU-AACGACGAC $5^{\prime}$
$\mathrm{E}$

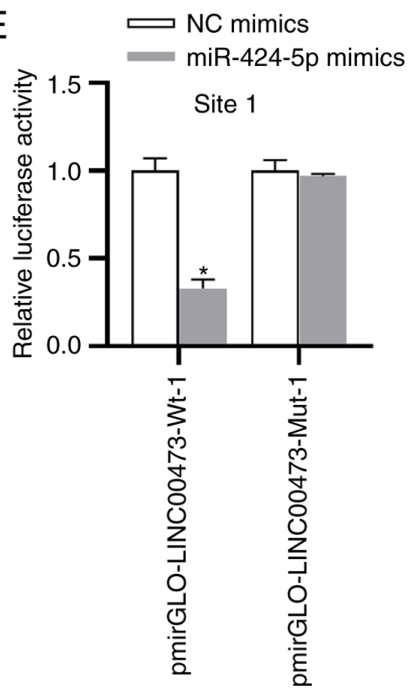

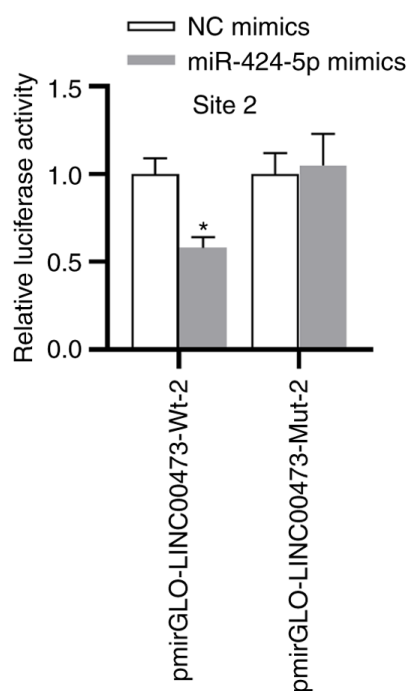

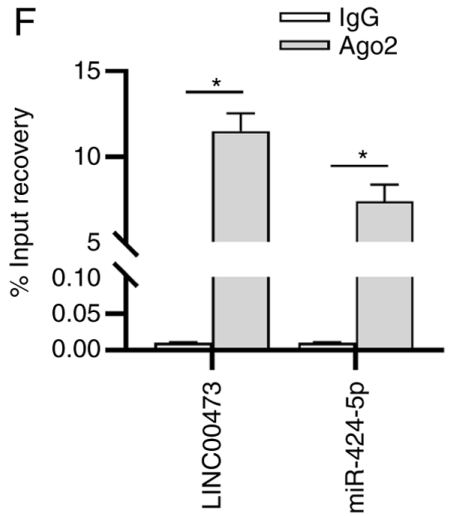

Figure 4. LINC00473 acts as a sponge for miR-424-5p. (A) Fluorescence in situ hybridization was conducted to examine the subcellular distribution of LINC00473 in IL-1 $\beta$-stimulated C28/I2 cells (magnification, x200 magnification; scale bar, $50 \mu \mathrm{m}$ ). (B) Reverse transcription-quantitative PCR was conducted to detect the relative expression levels of seven candidate miRs of LINC00473 in IL-1 $\beta$-stimulated C28/I2 cells. (C) Overexpression or knockdown efficacy of miR-424-5p in C28/I2 cells. (D) Binding sites between LINC00473 and miR-424-5p were predicted using the starBase database. Interaction between LINC00473 and miR-424-5p in C28/I2 cells was confirmed by (E) performing luciferase reporter and (F) RNA immunoprecipitation assays. "P<0.05. LINC00473, long intergenic non-protein coding RNA 473; miR, microRNA; Wt, wild-type; Mut, mutant; NC, negative control.

with the corresponding $\mathrm{NC}$ groups (Fig. 5E-H). As presented in Fig. 5I, LY6E expression in C28/I2 cells was significantly decreased by the transfection of si-LY6E compared with that of si-NC. The flow cytometry results indicated that IL- $1 \beta$ promoted the apoptosis of C28/I2 cells compared with the control group, and cell apoptosis was significantly suppressed in IL- $1 \beta+$ si-LY6E group compared with that in IL- $1 \beta+$ si-NC group (Fig. 5J and K). Furthermore, the concentrations of proinflammatory cytokines (IL-6, IL-8 and TNF- $\alpha$ ) were significantly elevated in IL-1 $\beta$-stimulated C28/I2 cells compared with the control cells; whereas these effects were partially reversed by LY6E knockdown (Fig. 5L-N).

LINC00473 exacerbates OA progression by regulating $L Y 6 E$. The follow-up rescue assays were performed to determine whether LINC00473 regulated LY6E to induce OA development. The overexpression efficacy of LY6E in IL-1 $\beta$-stimulated C28/I2 cells was verified by RT-qPCR. The 
A

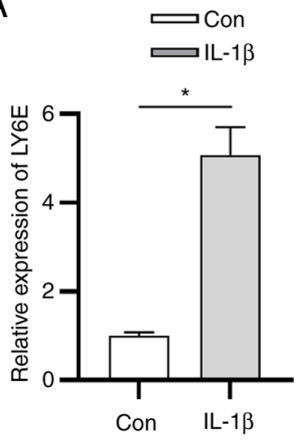

B

LY6E-Wt: $\quad 5^{\prime}$ cauuggagcccuccUGCUGCUu 3'

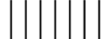

miR-424-5p: $\quad$ 3' aaguuuuguacuuaACGACGAc 5'

LY6E-Mut: $\quad 5^{\prime} \quad$ cauuggagcccucc ACGACGAu $3^{\prime}$

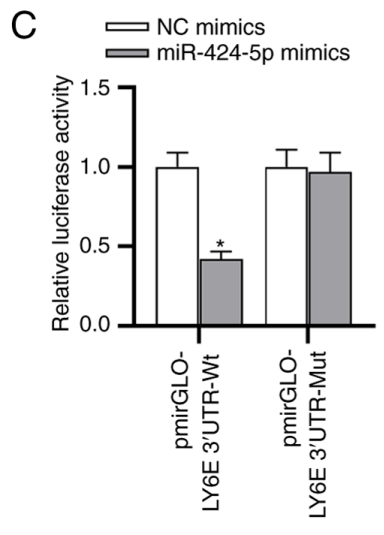

$\mathrm{H}$

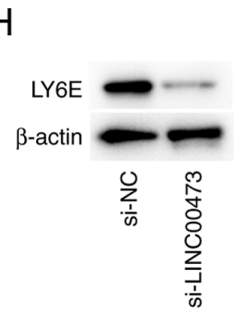

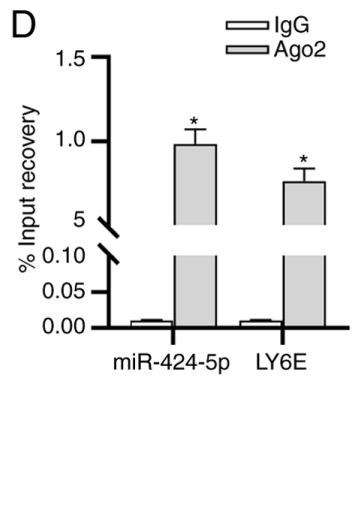

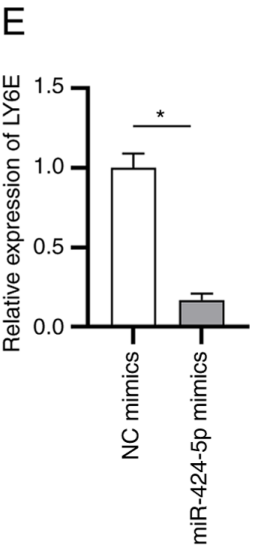

F

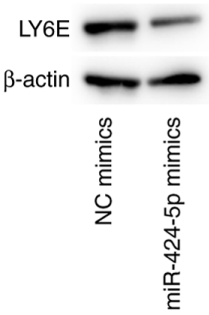

G

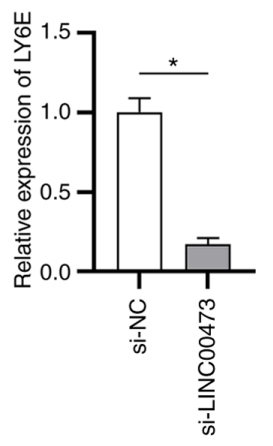

$J$

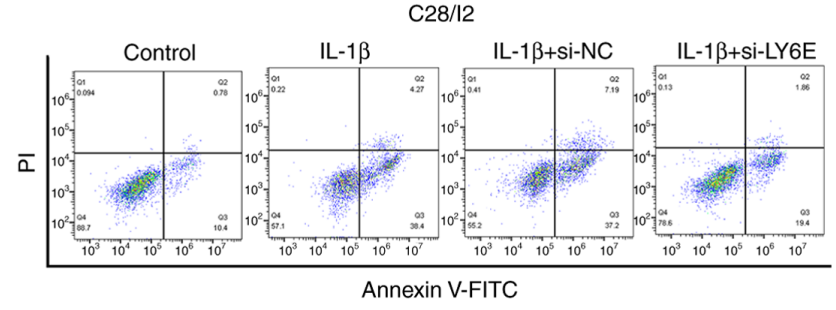

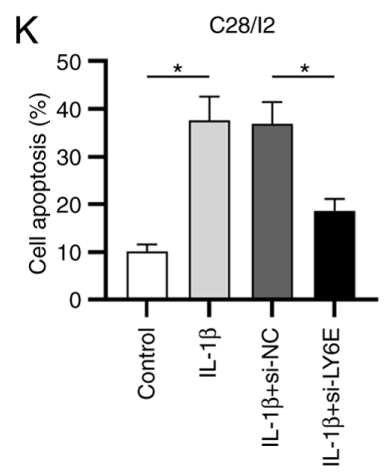
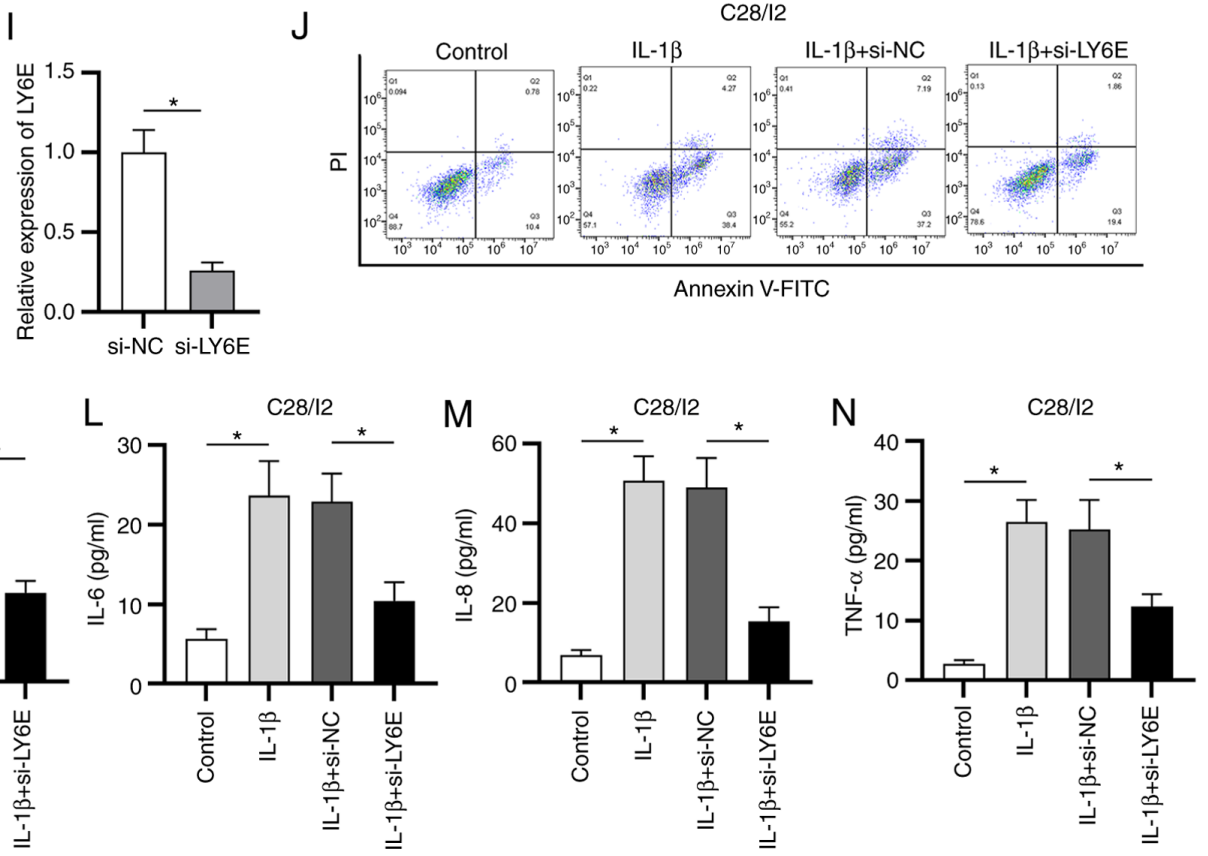

Figure 5. LY6E is a direct target of miR-424-5p. (A) RT-qPCR analysis was used to evaluate LY6E expression in IL-1 $\beta$-stimulated C28/I2 cells. The binding capacity between miR-424-5p and LY6E in C28/I2 cells was (B) predicted via starBase and (C) verified by performing the luciferase reporter assay. (D) Enrichment of miR-424-5p and LY6E in C28/I2 cells was measured using an RNA immunoprecipitation assay. Effects of miR-424-5p-overexpression on the (E) mRNA and (F) protein expression levels of LY6E in C28/I2 cells. (G) mRNA and (H) protein expression levels of LY6E were detected in si-LINC00473or si-NC-transfected C28/I2 cells using RT-qPCR and western blotting, respectively. (I) RT-qPCR analysis was conducted to evaluate the knockdown efficacy of si-LY6E in C28/I2 cells. ( $\mathrm{J}$ and K) Effect of LY6E knockdown on IL-1 $\beta$-induced apoptosis in C28/I2 cells was measured. ELISAs were used to determine the concentrations of inflammatory cytokines (L) IL-6, (M) IL-8 and (N) TNF- $\alpha$ in IL-1 $\beta$-stimulated C28/I2 cells. "P<0.05. LY6E, lymphocyte antigen 6 locus E; miR, microRNA; RT-qPCR, reverse transcription-quantitative PCR; si, small interfering RNA; LINC00473, long intergenic non-protein coding RNA 473; NC, negative control; Wt, wild-type; Mut, mutant; Con, control; PI, propidium iodide.

results revealed that the expression of LY6E was significantly elevated in C28/I2 cells transfected with pcDNA3.1/LY6E compared with the empty vector (Fig. 6A). As presented in
Fig. 6B and C, LINC00473 knockdown suppressed the apoptosis of IL-1 $\beta$-treated C28/I2 cells compared with the IL-1 $\beta+$ si-NC group, while LY6E overexpression partially reversed 
A
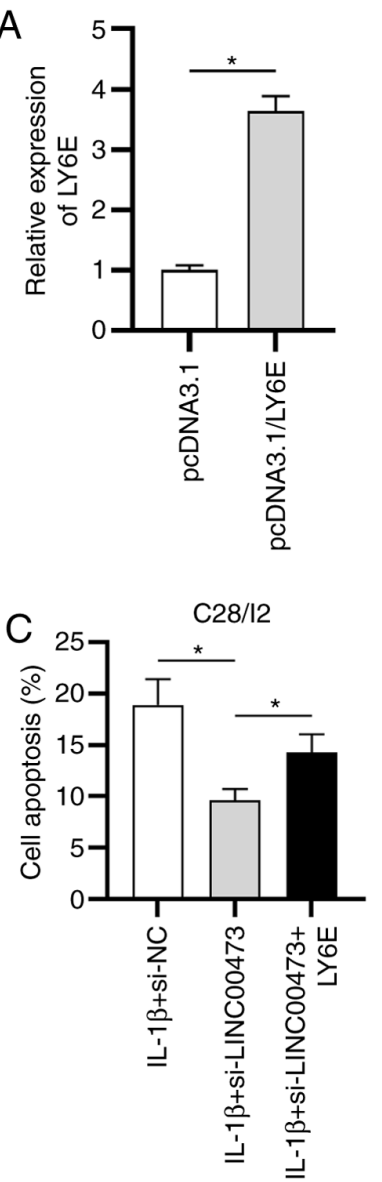

$\mathrm{F}$

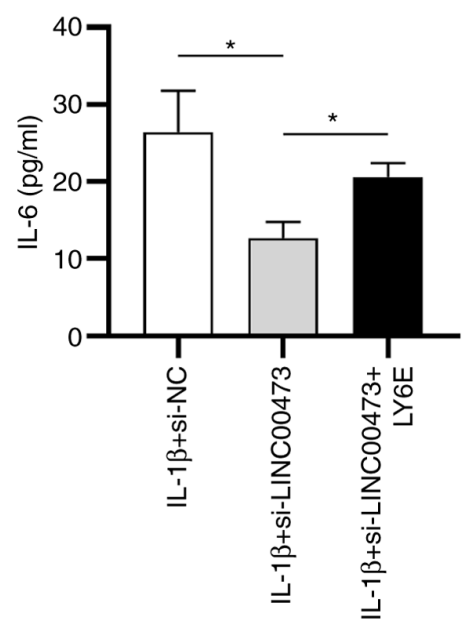

B

$\mathrm{D}$
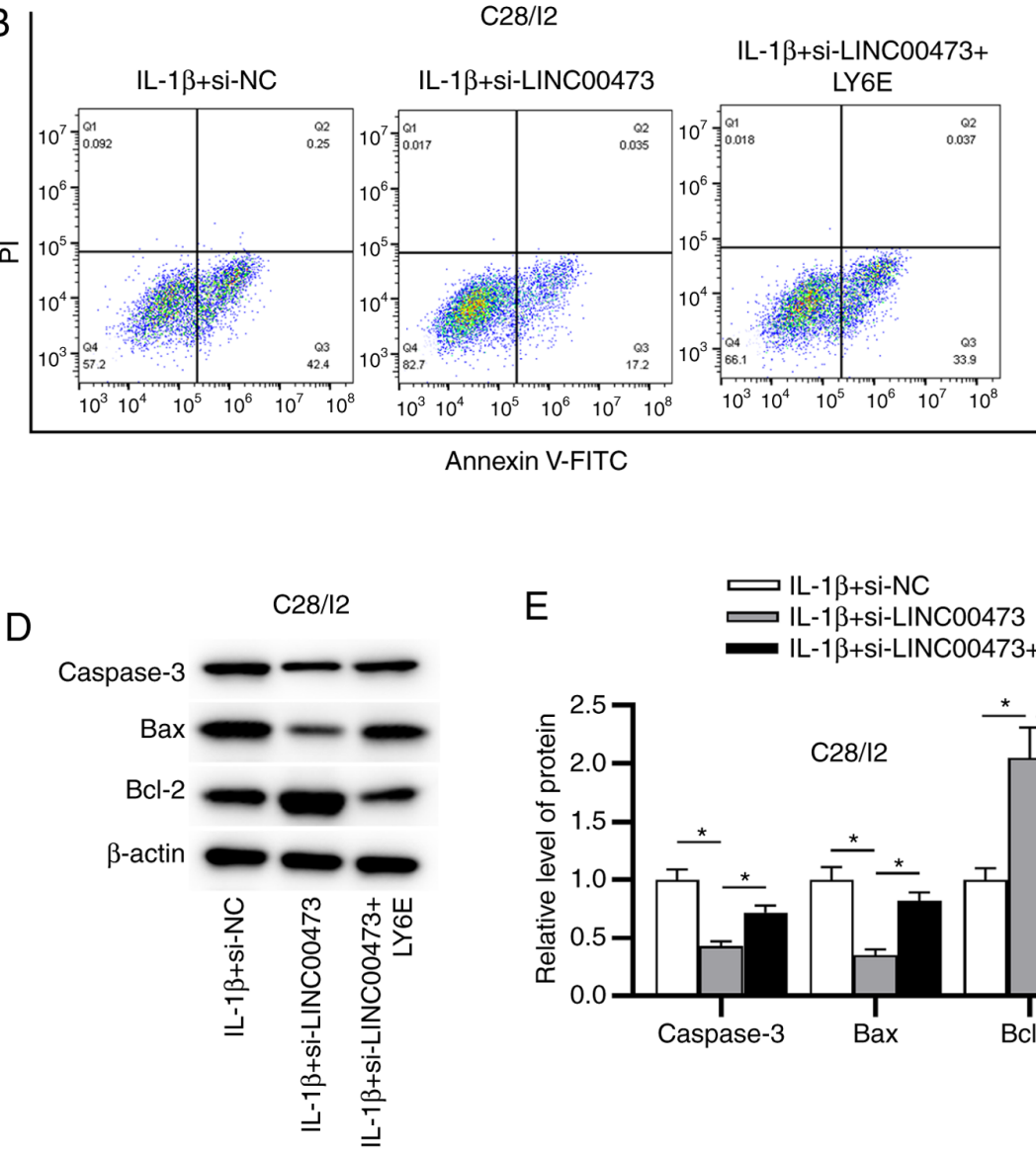

G

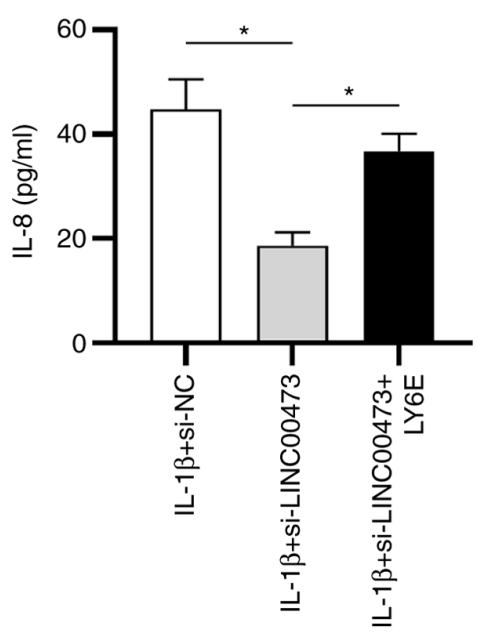

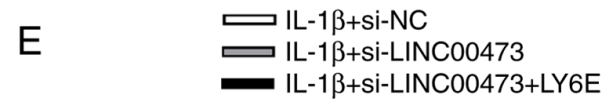

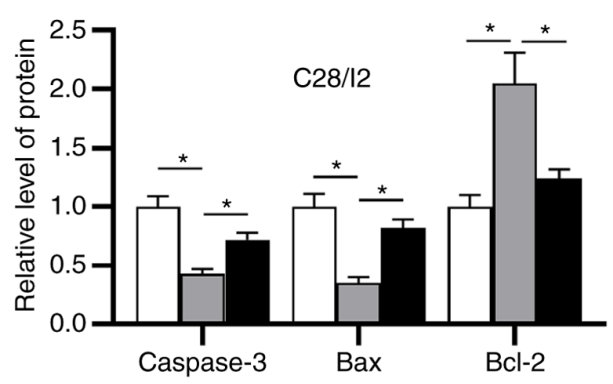

$\mathrm{H}$

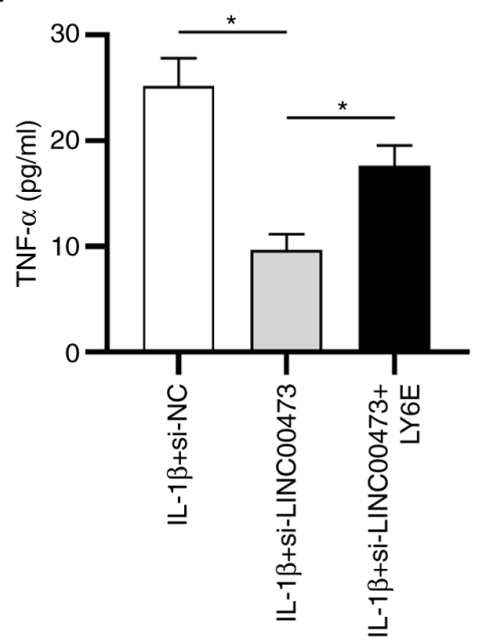

Figure 6. LINC00473 regulates IL-1 $\beta$-stimulated C28/I2 apoptosis and the inflammatory response by regulating LY6E. (A) Overexpression efficacy of LY6E in C28/I2 cells was determined using reverse transcription-quantitative PCR. (B and C) Flow cytometry was performed to assess apoptosis in transfected C28/I2 cells. Protein expression levels of caspase-3, Bax and Bcl-2 in C28/I2 cells under the indicated transfections were (D) detected by western blotting and (E) semi-quantified. Concentrations of inflammatory cytokines (F) IL-6, (G) IL-8 and (H) TNF- $\alpha$ were measured using ELISAs in transfected C28/I2 cells. "P<0.05. LINC00473, long intergenic non-protein coding RNA 473; LY6E, lymphocyte antigen 6 locus E; si, small interfering RNA; NC, negative control; PI, propidium iodide

LINC00473 knockdown-mediated inhibitory effect on the apoptosis of IL-1 $\beta$-treated C28/I 2 cells. In addition, the protein expression levels of caspase-3 and Bax were significantly decreased in the IL-1 $\beta+$ si-LINC00473 group compared with the si-NC, whereas Bcl-2 expression was significantly increased. However, these effects were significantly offset by LY6E overexpression in IL-1 $\beta$-stimulated C28/I2 cells (Fig. 6D and E). Furthermore, the results demonstrated that LY6E overexpression partially reversed the suppressive effects of LINC00473 knockdown on the production of inflammatory cytokines (IL-6, IL- 8 and TNF- $\alpha$ ) by IL-1 $\beta$-stimulated C28/I2 cells (Fig. 6F-H). 


\section{Discussion}

OA is a chronic disease that is characterized by several features, such as cartilage degradation and synovial inflammation (30). The cumulative incidence of symptomatic knee OA among middle-aged and older Chinese adults was $8.5 \%$, and the incidence among females $(11.2 \%)$ was higher than that among males $(5.6 \%)$ based on data collected from China Health and Retirement Longitudinal Study, which is a national population survey with a 4-year follow-up, between 2011 and 2015 (36). Over the next few decades, the incidence of OA is expected to continuously rise, which may have a significant impact on the daily activities of individuals (37). Current medical therapy of OA primarily focuses on pain relief (38). However, there are still a number of challenges, including the lack of disease-modifying drugs, therapeutic efficacy of anti-inflammatory drugs and the safety of new therapies, in the treatment of OA (39).

It is known that lncRNAs are non-coding segments of RNAs that have notable regulatory functions in the pathophysiological processes of multiple diseases, including OA (13-15). LINC00473, a novel lncRNA, has been revealed to be upregulated in several diseases, such as ischemic stroke and preeclampsia $(16,17)$. Moreover, LINC00473 is upregulated in patients with severe pain associated with OA (18). Consistent with the high expression of LINC00473 in ischemic stroke (16), preeclampsia (17) and patients diagnosed with OA (18), the current study revealed that LINC00473 was highly expressed in the cartilage tissue of patients with $\mathrm{OA}$, in IL-1 $\beta$-treated C28/I2 cells and in the cartilage tissues of OA model rats.

Accumulating evidence has suggested that the pathogenesis of OA is complex and is usually associated with chondrocyte apoptosis, inflammation and ECM degeneration (40,41). For example, hypoxia-inducible factor- $2 \alpha$ aggravated cartilage destruction by enhancing chondrocyte apoptosis in OA (42). Furthermore, cartilage injury-related lncRNAs facilitated the degradation of ECM in OA progression (43). MALAT1 also regulated the inflammation of synovial fibroblasts in obese patients and those with OA (44). Thus, further determining the mechanism of LINC00473 may be useful to elucidate a novel therapeutic strategy for OA. The present study revealed that LINC00473 knockdown efficiently alleviated OA development in vitro and in vivo by decreasing chondrocyte apoptosis, degradation of the ECM and production of proinflammatory cytokines.

miRNAs are a series of endogenous small non-coding RNAs that have been reported to be involved in numerous diseases (45). For example, miR-155 exacerbated pulmonary inflammation induced by cigarette smoke and the development of chronic obstructive pulmonary disease (46). miR-182 served a protective role in hypoxia-induced cardiomyocytes by targeting hes family bHLH transcription factor 1 (47). Additionally, miR-30a served as a virulence miRNA in OA development by promoting ECM degradation (48). Emerging evidence has revealed that miRNAs exert their biological functions by interacting with lncRNAs in OA (49-51). In the present study, two binding sites between miR-424-5p and LINC00473 were predicted by bioinformatics analysis. The binding ability between them was subsequently verified, indicating that
LINC00473 functioned as a sponge for miR-424-5p in C28/I2 cells. Furthermore, a number of studies have demonstrated that miRNAs modulate the expression levels of specific genes in diseases $(47,52,53)$.

As a key member of the lymphostromal cell membrane Ly6 protein superfamily, LY6E serves a notable role in immunological regulation and oncogenesis (54). Previous studies have suggested that LY6E participates in the progression of several diseases (55-57). For example, LY6E expression could function as a biomarker in the diagnosis of systemic lupus erythematosus $(55,56)$. Using RNA sequencing, LY6E was also revealed to be involved in ischemic stroke (57). However, to the best of our knowledge, the role of LY6E has not been identified in OA. The present study revealed that miR-424-5p had binding sites on the 3'UTR of LY6E and further proved the binding capability between miR-424-5p and LY6E. Moreover, LY6E knockdown significantly inhibited IL-1 $\beta$-induced apoptosis and proinflammatory cytokine production in C28/I2 cells. In addition, rescue experiments revealed that LY6E overexpression significantly reversed the inhibitory effects of LINC00473 knockdown on chondrocyte apoptosis and proinflammatory cytokine production. These data indicated that LINC00473 may be involved in OA pathogenesis via dysregulated ceRNA interactions.

In summary, the present study revealed that LINC00473 knockdown suppressed IL-1 $\beta$-induced apoptosis and production of proinflammatory cytokines in C28/I2 cells via the miR-424-5p/LY6E axis. The results may provide a novel perspective for the improved understanding of the pathogenesis of OA, and may also improve the treatment of OA.

\section{Acknowledgements}

Not applicable.

\section{Funding}

No funding was received.

\section{Availability of data and materials}

The datasets used and/or analyzed during the current study are available from the corresponding author on reasonable request.

\section{Authors' contributions}

GF and XG designed the research. GF and JL performed the research. GF and YZ analyzed the data. GF and XG wrote the paper. GF and XG confirm the authenticity of all the raw data. All authors read and approved the final manuscript.

\section{Ethics approval and consent to participate}

The current study was approved by The Human Ethics Committee of Children's Hospital of Soochow University (Jiangsu, China; approval number, KS20190036). Informed consent was obtained from all participants included in the current study. All procedures performed in studies involving human participants were in accordance with the ethical 
standards of the institutional and/or national research committee and with the 1964 Declaration of Helsinki and its later amendments or comparable ethical standards. The animal experiments were approved by The Attitude of the Animal Care \& Welfare Committee of Children's Hospital of Soochow University (Jiangsu, China; approval no. 2019-044).

\section{Patient consent for publication}

Not applicable.

\section{Competing interests}

The authors declare that they have no competing interests.

\section{References}

1. Palazzo C, Nguyen C, Lefevre-Colau MM, Rannou F and Poiraudeau S: Risk factors and burden of osteoarthritis. Ann Phys Rehabil Med 59: 134-138, 2016.

2. Liu-Bryan R: Inflammation and intracellular metabolism: New targets in OA. Osteoarthritis Cartilage 23: 1835-1842, 2015.

3. Maldonado M and Nam J: The role of changes in extracellular matrix of cartilage in the presence of inflammation on the pathology of osteoarthritis. Biomed Res Int 2013: 284873, 2013.

4. Perez Martin A: Symptoms. Localizations: Knee, hip, hands, spine, other localizations. Aten Primaria 46 (Suppl 1): S11-S17, 2014 (In Spanish).

5. Loeser RF, Goldring SR, Scanzello CR and Goldring MB: Osteoarthritis: A disease of the joint as an organ. Arthritis Rheum 64: 1697-1707, 2012.

6. Majeed MH, Sherazi SAA, Bacon D and Bajwa ZH: Pharmacological treatment of pain in osteoarthritis: A descriptive review. Curr Rheumatol Rep 20: 88, 2018.

7. Xu YK, Zhu FG, Feng EY, Fan CL, Yu GS, Wei YZ and Xie LH: External therapies of traditional Chinese medicine combined with sodium hyaluronate injected in articular cavity therapy on knee osteoarthritis: Meta-analysis. Zhongguo Zhong Yao Za Zhi 43: 1934-1939, 2018 (In Chinese).

8. Richards MM, Maxwell JS, Weng L, Angelos MG and Golzarian J: Intra-articular treatment of knee osteoarthritis: From anti-inflammatories to products of regenerative medicine. Phys Sportsmed 44: 101-108, 2016.

9. Paraskevopoulou MD and Hatzigeorgiou AG: Analyzing MiRNA-LncRNA interactions. Methods Mol Biol 1402: 271-286, 2016.

10. Kazemzadeh M, Safaralizadeh R and Orang AV: LncRNAs: Emerging players in gene regulation and disease pathogenesis. J Genet 94: 771-784, 2015.

11. Mathieu EL, Belhocine M, Dao LT, Puthier D and Spicuglia S: Functions of lncRNA in development and diseases. Med Sci (Paris) 30: 790-796, 2014 (In French).

12. Xie F, Liu YL, Chen XY, Li Q, Zhong J, Dai BY, Shao XF and Wu GB: Role of MicroRNA, LncRNA, and exosomes in the progression of osteoarthritis: A review of recent literature. Orthop Surg 12: 708-716, 2020.

13. Zhou Q, Huang XR, Yu J, Yu X and Lan HY: Long noncoding RNA Arid2-IR is a novel therapeutic target for renal inflammation. Mol Ther 23: 1034-1043, 2015.

14. Cai L, Tu L, Li T, Yang X, Ren Y, Gu R, Zhang Q, Yao H, $\mathrm{Qu}$ X, Wang Q and Tian J: Downregulation of lncRNA UCA1 ameliorates the damage of dopaminergic neurons, reduces oxidative stress and inflammation in Parkinson's disease through the inhibition of the PI3K/Akt signaling pathway. Int Immunopharmacol 75: 105734, 2019.

15. Zhang Y, Wang F, Chen G, He R and Yang L: LncRNA MALAT1 promotes osteoarthritis by modulating miR-150-5p/AKT3 axis. Cell Biosci 9: 54, 2019.

16. Zheng Y, Sun S, Yu M and Fu X: Identification of potential hub-lncRNAs in ischemic stroke based on Subpathway-LNCE method. J Cell Biochemistry 120: 12832-12842, 2019.
17. Wu D, Xu Y, Zou Y, Zuo Q, Huang S, Wang S, Lu X, He X, Wang J, Wang T and Sun L: Long noncoding RNA 00473 is involved in preeclampsia by LSD1 binding-regulated TFPI2 transcription in trophoblast cells. Mol Ther Nucleic Acids 12: 381-392, 2018.

18. Chen Y, Lin Y, Bai Y, Cheng D and Bi Z: A long noncoding RNA (lncRNA)-associated competing endogenous RNA (ceRNA) network identifies eight lncRNA biomarkers in patients with osteoarthritis of the knee. Med Sci Monit 25: 2058-2065, 2019.

19. Salmena L, Poliseno L, Tay Y, Kats L and Pandolfi PP: A ceRNA hypothesis: The Rosetta Stone of a hidden RNA language? Cell 146: 353-358, 2011.

20. Kong F, Jin J, Lv X, Han Y, Liang X, Gao Y and Duan X: Long noncoding RNA RMRP upregulation aggravates myocardial ischemia-reperfusion injury by sponging miR-206 to target ATG3 expression. Biomed Pharmacother 109: 716-725, 2019.

21. Li X, Zeng L, Cao C, Lu C, Lian W, Han J, Zhang X, Zhang J, Tang $\mathrm{T}$ and $\mathrm{Li} \mathrm{M}$ : Long noncoding RNA MALAT1 regulates renal tubular epithelial pyroptosis by modulated miR-23c targeting of ELAVL1 in diabetic nephropathy. Exp Cell Res 350: 327-335, 2017.

22. Shen H, Wang Y, Shi W, Sun G, Hong L and Zhang Y: LncRNA SNHG5/miR-26a/SOX2 signal axis enhances proliferation of chondrocyte in osteoarthritis. Acta Biochim Biophys Sin (Shanghai) 50: 191-198, 2018

23. Glyn-Jones S, Palmer AJ, Agricola R, Price AJ, Vincent TL, Weinans H and Carr AJ: Osteoarthritis. Lancet 386: 376-387, 2015.

24. National Research Council (US) Committee for the Update of the Guide for the Care and Use of Laboratory Animals: Guide for the Care and Use of Laboratory Animals. 8th edition. Washington, DC, National Academies Press (US), 2011.

25. Livak KJ and Schmittgen TD: Analysis of relative gene expression data using real-time quantitative PCR and the 2(-Delta Delta C(T)) method. Methods 25: 402-408, 2001.

26. Wang Y, Shen S, Li Z, Li W and Weng X: MIR-140-5p affects chondrocyte proliferation, apoptosis, and inflammation by targeting HMGB1 in osteoarthritis. Inflamm Res 69: 63-73, 2020.

27. Cheng C, Shan W, Huang W, Ding Z, Cui G, Liu F, Lu W, Xu J, He W and Yin Z: ACY-1215 exhibits anti-inflammatory and chondroprotective effects in human osteoarthritis chondrocytes via inhibition of STAT3 and NF- $\mathrm{KB}$ signaling pathways. Biomed Pharmacother 109: 2464-2471, 2019.

28. Wang Y, Yu T, Jin H, Zhao C and Wang Y: Knockdown MiR-302b alleviates LPS-induced injury by targeting Smad3 in C28/I2 chondrocytic cells. Cell Physiol Biochem 45: 733-743, 2018.

29. Fernandes JC, Martel-Pelletier J and Pelletier JP: The role of cytokines in osteoarthritis pathophysiology. Biorheology 39: 237-246, 2002.

30. Charlier E, Deroyer C, Ciregia F, Malaise O, Neuville S, Plener Z, Malaise M and de Seny D: Chondrocyte dedifferentiation and osteoarthritis (OA). Biochem Pharmacol 165: 49-65, 2019.

31. Goldring MB and Otero M: Inflammation in osteoarthritis. Curr Opin Rheumatol 23: 471-478, 2011.

32. Zhang Y, Lin J, Zhou X, Chen X, Chen AC, Pi B, Pan G, Pei M, Yang H, Liu T and He F: Melatonin prevents osteoarthritis-induced cartilage degradation via targeting MicroRNA-140. Oxid Med Cell Longev 2019: 9705929, 2019.

33. Zhang P, Sun J, Liang C, Gu B, Xu Y, Lu H, Cao B and Xu H: lncRNA IGHC $\gamma$ Acts as a ceRNA to regulate macrophage inflammation via the miR-6891-3p/TLR4 axis in osteoarthritis. Mediators Inflamm 2020: 9743037, 2020.

34. Chen $\mathrm{H}$ and Chen L: An integrated analysis of the competing endogenous RNA network and co-expression network revealed seven hub long non-coding RNAs in osteoarthritis. Bone Joint Res 9: 90-98, 2020.

35. Tay Y, Rinn J and Pandolfi PJN: The multilayered complexity of ceRNA crosstalk and competition. Nature 505: 344-352, 2014.

36. Ren Y, Hu J, Tan J, Tang X, Li Q, Yang H, Liu C, He Q, Zou K, Sun $\mathrm{X}$ and Tan B: Incidence and risk factors of symptomatic knee osteoarthritis among the Chinese population: Analysis from a nationwide longitudinal study. BMC Public Health 20: 1491, 2020.

37. Callahan LF, Ambrose KR, Albright AL, Altpeter M, Golightly YM, Huffman KF, Nelson AE and Weisner SE: Public Health interventions for osteoarthritis-updates on the osteoarthritis Action Alliance's efforts to address the 2010 OA Public Health Agenda Recommendations. Clin Exp Rheumatol 37 (Suppl 120): S31-S39, 2019. 
38. Vargas Negrin F, Medina Abellan MD, Hermosa Hernan JC and de Felipe Medina R: Treatment of patients with osteoarthritis. Aten Primaria 46 (Suppl 1): S39-S61, 2014 (In Spanish).

39. Hermann W, Lambova S and Muller-Ladner U: Current treatment options for osteoarthritis. Curr Rheumatol Rev 14: 108-116, 2018.

40. Hwang HS and Kim HA: Chondrocyte apoptosis in the pathogenesis of osteoarthritis. Int J Mol Sci 16: 26035-26054, 2015.

41. Minguzzi M, Cetrullo S, D'Adamo S, Silvestri Y, Flamigni F and Borzi RM: Emerging players at the intersection of chondrocyte loss of maturational arrest, oxidative stress, senescence and low-grade inflammation in osteoarthritis. Oxid Med Cel Longev 2018: 3075293, 2018.

42. Ryu JH, Shin Y, Huh YH, Yang S, Chun CH and Chun JS Hypoxia-inducible factor-2alpha regulates Fas-mediated chondrocyte apoptosis during osteoarthritic cartilage destruction. Cell Death Differ 19: 440-450, 2012.

43. Liu Q, Zhang X, Dai L, Hu X, Zhu J, Li L, Zhou C and Ao Y: Long noncoding RNA related to cartilage injury promotes chondrocyte extracellular matrix degradation in osteoarthritis. Arthritis Rheumatol 66: 969-978, 2014.

44. Nanus DE, Wijesinghe SN, Pearson MJ, Hadjicharalambous MR, Rosser A, Davis ET, Lindsay MA and Jones SW: Obese osteoarthritis patients exhibit an inflammatory synovial fibroblast phenotype, which is regulated by the long non coding RNA MALAT1. Arthritis Rheumatol 72: 609-619, 2019.

45. Beermann J, Piccoli MT, Viereck J and Thum T: Non-coding RNAs in development and disease: Background, mechanisms, and therapeutic approaches. Physiol Rev 96: 1297-1325, 2016.

46. De Smet EG, Van Eeckhoutte HP, Avila Cobos F, Blomme E, Verhamme FM, Provoost S, Verleden SE, Venken K, Maes T, Joos GF, et al: The role of miR-155 in cigarette smoke-induced pulmonary inflammation and COPD. Mucosal Immunol 13: 423-436, 2020

47. Zhang Y, Peng B and Han Y: MiR-182 alleviates the development of cyanotic congenital heart disease by suppressing HES1. Eur J Pharmacol 836: 18-24, 2018.

48. Chang T, Xie J, Li H, Li D, Liu P and Hu Y: MicroRNA-30a promotes extracellular matrix degradation in articular cartilage via downregulation of Sox9. Cell Prolif 49: 207-218, 2016.
49. Zhang G, Wu Y, Xu D and Yan X: Long Noncoding RNA UFC1 promotes proliferation of chondrocyte in osteoarthritis by acting as a sponge for miR-34a. DNA Cell Biol 35: 691-695, 2016.

50. Li Y, Li S, Luo Y, Liu Y and Yu N: LncRNA PVT1 regulates chondrocyte apoptosis in osteoarthritis by acting as a sponge for miR-488-3p. DNA Cell Biol 36: 571-580, 2017.

51. Li YF, Li SH, Liu Y and Luo YT: Long noncoding RNA CIR promotes chondrocyte extracellular matrix degradation in osteoarthritis by acting as a sponge For Mir-27b. Cell Physiol Biochem 43: 602-610, 2017.

52. Pan W, Yu H, Zheng B, Gao Y, Li P, Huang Q, Xie C and Ge X: Upregulation of MiR-369-3p suppresses cell migration and proliferation by targeting SOX4 in Hirschsprung's disease. J Pediatr Surg 52: 1363-1370, 2017.

53. Chen L, Li Q, Wang J, Jin S, Zheng H, Lin J, He F, Zhang H, Ma S, Mei J and Yu J: MiR-29b-3p promotes chondrocyte apoptosis and facilitates the occurrence and development of osteoarthritis by targeting PGRN. J Cell Mol Med 21: 3347-3359, 2017.

54. Yu J and Liu SL: Emerging role of LY6E in virus-host interactions. Viruses 11: 1020, 2019.

55. Feng X, Huang J, Liu Y, Xiao L, Wang D, Hua B, Tsao BP and Sun L: Identification of interferon-inducible genes as diagnostic biomarker for systemic lupus erythematosus. Clin Rheumatol 34: 71-79, 2015

56. Komatsuda A, Wakui H, Iwamoto K, Ozawa M, Togashi M, Masai R, Maki N, Hatakeyama T and Sawada K: Up-regulated expression of Toll-like receptors mRNAs in peripheral blood mononuclear cells from patients with systemic lupus erythematosus. Clin Exp Immunol 152: 482-487, 2008.

57. He W, Wei D, Cai, Chen S, Li S and Chen W: Altered long non-coding RNA Transcriptomic Profiles in Ischemic Stroke. Hum Gene Ther 29: 719-732, 2018.

This work is licensed under a Creative Commons Attribution-NonCommercial-NoDerivatives 4.0 International (CC BY-NC-ND 4.0) License. 